\title{
A INFLUÊNCIA DA ÁREA URBANA SOBRE A QUALIDADE DA ÁGUA NA BACIA HIDROGRÁFICA DO RIBEIRÃO DOS POÇOS (POÇOS DE CALDAS, MG)
}

\section{THE URBAN AREA INFLUENCE ON WATER QUALITY OF RIBEIRÃO DOS POÇOS WATERSHED (POÇOS DE CALDAS, MG)}

\section{Antonio Donizetti Gonçalves de Souza, Amauri Henrique de Souza}

\author{
Universidade Federal de Alfenas/Instituto de Ciência e Tecnologia. Rua Gabriel \\ Monteiro da Silva, 714 - Centro, Alfenas - MG, 37130-000. E-mail \\ adonizetti@unifal-mg.edu.br
}

\begin{abstract}
RESUMO
O presente estudo teve como objetivo realizar a avaliação das influências da área urbanizada sobre a qualidade da água na bacia hidrográfica do Ribeirão dos Poços (Poços de Caldas-MG), visando subsidiar instrumentos de gestão dos recursos hídricos. Para o estudo foram considerados os aspectos das interferências humanas na bacia, o meio físico, uso dos recursos hídricos e fontes poluidoras. A qualidade da água foi avaliada nos dois períodos sazonais (inverno e verão), com frequência intensiva (seis coletas diárias) em 06 (seis) sítios representativos, medindo-se os fatores: temperatura $\left({ }^{\circ} \mathrm{C}\right), \mathrm{pH}$, turbidez (NTU), condutividade $\left(\mathrm{S} . \mathrm{cm}^{-1}\right)$, oxigênio dissolvido $\left(\mathrm{mg} . \mathrm{L}^{-1}\right)$,

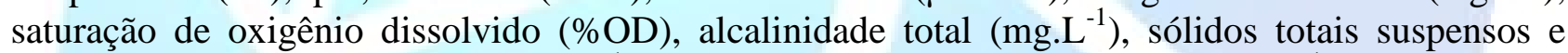
frações orgânicas e inorgânicas $\left(\mathrm{mg} . \mathrm{L}^{-1}\right)$ e carga de sólidos totais suspensos (kg.dia $\left.{ }^{-1}\right)$. Os resultados obtidos indicaram forte influência da área urbana sobre o corpo de água, registrando valores mínimos de oxigênio dissolvido e gradientes de aumento da condutividade, turbidez e sólidos em estações localizadas na área urbana. A sazonalidade também demonstrou influência sobre a qualidade da água indicando níveis mais altos de oxigenação e turbidez no período chuvoso em comparação com período de seca. De forma geral, os resultados demonstraram que a bacia ao atravessar a área urbana sofre impactos pontuais e difusos que necessitam ser mais bem estudados para tomadas de medidas corretivas e preventivas.

Palavra-chave: Bacia hidrográfica. Qualidade da água. Recursos hídricos. Gestão de recursos hídricos.
\end{abstract}

\footnotetext{
ABSTRACT

This study aimed to perform assessment of the influences of the urban area on the water quality in the watershed of Ribeirão dos Poços (Poços de Caldas-MG) to subsidize instruments to manage water resources. For the study were collected and aspects of human interference in the bowl, physiography of the drainage network, physical environment, use of water resources and pollution sources. The water quality was evaluated in both seasonal periods (winter and summer) with intensive frequency (six daily collections) in six (06) representative sites by measuring the
} 
following factores: temperature $\left({ }^{\circ} \mathrm{C}\right), \mathrm{pH}$, turbidity (NTU), conductivity ( $\left.\mathrm{S} . \mathrm{cm}-1\right)$, dissolved oxygen $\left(\mathrm{mg} \mathrm{L}^{-1}\right)$, saturation dissolved oxygen $(\%)$, total alkalinity $\left(\mathrm{mg} . \mathrm{L}^{-1}\right)$, total suspended solids, organic and inorganic ( $\mathrm{mg}$ fractions. $\mathrm{L}^{-1}$ ) counts of total suspended solids $\left(\mathrm{kg}^{-\mathrm{day}}{ }^{-1}\right)$. These results indicate a strong influence of urban areas on the water body, recording minimum values of dissolved oxygen gradients and increased conductivity, turbidity and solids at stations located in the urban area. Seasonality also demonstrated influence on water quality indicating higher levels of oxygenation and turbidity during the rainy season compared to the dry season. Overall, the results demonstrated that the basin to cross the urban area suffers punctual and diffuse impacts that need to be further studied for taking corrective and preventive measures.

Keyword: Watershed. Water quality. Water resources. Management of water resources.

\section{INTRODUÇÃO}

A demanda de água e a progressiva deterioração de sua qualidade têm despertado a necessidade do desenvolvimento de ações integradas de planejamento e gerenciamento visando a diminuição dos conflitos de uso.

Para Rocha et al. (2000), o uso da bacia hidrográfica como unidade de planejamento nas investigações e no gerenciamento dos recursos hídricos originou-se da percepção de que os ecossistemas aquáticos são essencialmente abertos, trocam matéria e energia entre si e, com os ecossistemas terrestres adjacentes. Ao mesmo tempo, eles sofrem diferentes tipos alterações em virtude dos usos do solo e das atividades antropogênicas nele desenvolvidas. No caso de sistemas lóticos interligados, como as redes de drenagem, as trocas são realizadas de acordo com o gradiente fisiográfico característico da bacia (ESPÍNDOLA et al., 2000).

O solo, água e vegetação não podem ser satisfatoriamente explorados se forem encarados de maneira isolada e independente um do outro. Reconhecer a bacia como um sistema interrelacionado e uma unidade de planejamento é necessário, pois, ela contém fatores indispensáveis ao manejo integrado (HUFSCHMIDT e McCAULEY, 1991; BRIGANTE e ESPÍNDOLA, 2003; TUNDISI, 2005).

A realização de diagnósticos ambientais que tenham como elemento integrador a qualidade da água em bacias hidrográficas em áreas urbanas assume um papel norteador, tanto para os estudos dos processos e geração de conhecimentos como para subsidiar os instrumentos de gestão.

Estudos integrados em bacias hidrográficas urbanas que tiveram como base estabelecer uma relação quantitativa entre a qualidade da água e os atributos ambientais da área foram realizados por Maasdam et al. (1994) e Vega et al. (1998), Eyre e Pepperell (1999) e Freitas et al. (2011). Esses autores aplicaram o conceito sistêmico de bacia hidrográfica para a avaliação da qualidade da água.

Os estudos em pequenas bacias têm mostrado de forma mais clara a dependência da qualidade da água em relação ao contorno terrestre e seu grau de conservação (MARGALEF, 1983; TUCCI e CLARKE, 1997). Para Meybeck (1989), a concentração de muitos elementos em rios é altamente variável em bacias menores que são mais facilmente influenciadas por fatores simples como o desmatamento de uma pequena área, ao passo que nas grandes redes de drenagem, a qualidade da água é geralmente menos influenciada e variável.

A poluição de corpos hídricos superficiais é um dos maiores problemas ambientais em todo o mundo, causando efeitos negativos para a saúde humana e ambiental, muitas vezes influenciando e prejudicando suas condições básicas de qualidade da água para seus diversos usos. Uma das principais causas para este tipo de poluição se deve ao lançamento de esgotos domésticos e industriais.

Segundo Moraesa e Jordão (2002), estima-se que cerca de $80 \%$ de todas as moléstias e mais de um terço dos óbitos dos países em desenvolvimento sejam causados pelo consumo de água 
contaminada. Cerca de 34.000 pessoas morrem diariamente de doenças relacionadas com a água como diarreia. No Brasil, $65 \%$ das internações hospitalares são devidas a doenças veiculadas pela água (TUCCI, 2003). Dessa forma, a preocupação com a degradação e consequente escassez dos recursos hídricos deixou de ser um problema para os ambientalistas e passou a representar um sério problema de saúde pública (MORAESA e JORDÃO, 2002).

A bacia em estudo atravessa toda a área urbana de Poços de Caldas recebendo lançamentos de esgotos domésticos e industriais. Os impactos gerados neste corpo de água também se manifestam à jusante, num dos principais sistemas hídricos da região, o Ribeirão das Antas, afluente do Rio Pardo. Estudos nesta bacia têm elevada importância, pois o entendimento dos processos e impactos para a tomada de decisão referente às medidas de prevenção e/ou correção serão mais efetivas para a melhoria da qualidade ambiental no Ribeirão das Antas e Rio Pardo a jusante. Além disto, a bacia representa um dos mananciais de abastecimento do município de Poços de Caldas.

O presente estudo teve como objetivo realizar a avaliação das influências da área urbanizada sobre a qualidade da água na bacia hidrográfica do Ribeirão dos Poços (Poços de Caldas-MG). O trabalho coloca como hipótese que a ocupação urbana causa perda da qualidade da água na bacia em estudo.

\section{MATERIAL E MÉTODOS}

A bacia hidrográfica do Ribeirão dos Poços localiza-se totalmente nos limites do município de Poços de Caldas - MG (46 $00^{\prime}-46^{\circ} 37^{\prime} \mathrm{W}$ e $\left.21^{\circ} 47^{\prime}-21^{\circ} 52^{\prime} \mathrm{S}\right)$. A Figura 1 apresenta a bacia do Ribeirão dos Poços e suas principais sub-bacias. A sub-bacia do córrego da Ponte Alta é utilizada como manancial do município por meio da captação existente na represa Saturnino de Brito. O meio físico da área está inserido em uma grande unidade geomorfológica denominada "Planaltos Dissecados do Sul de Minas" que se estende por toda a Região de Planejamento do Sul de Minas e adentra o Estado de São Paulo (COSTA, 2001; FERNANDES et al., 2003).

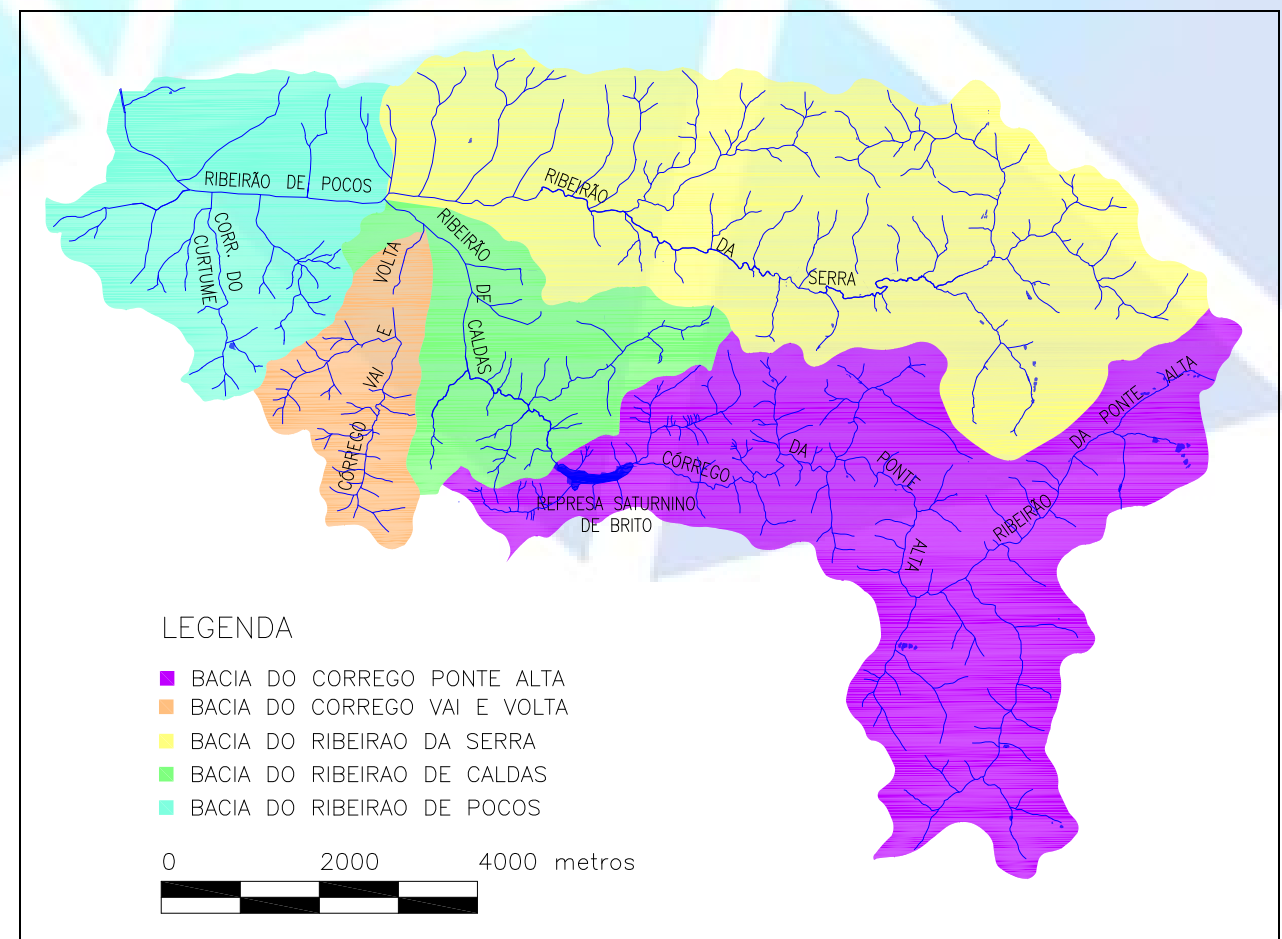

Figura 1. Bacia do Ribeirão dos Poços e suas principais sub-bacias. Fonte: Secretaria de Planejamento Prefeitura Municipal de Poços de Caldas (2003). 
O clima de Poços de Caldas, segundo a classificação de Köppen, é do tipo Cwb mesotérmico com inverno seco e verão brando. Este é marcado pela ocorrência de duas estações distintas: O verão chuvoso (outubro a março), caracterizado pelas temperaturas e precipitações elevadas (temperatura média de $20,3{ }^{\circ} \mathrm{C}$ e total no período de $1.430 \mathrm{~mm}$ de chuvas) e o inverno, seco (abril a setembro) marcado por temperaturas e índices pluviométricos baixos (temperatura média de $15^{\circ} \mathrm{C}$ e $315 \mathrm{~mm}$ de chuvas no período). A precipitação média anual em Poços de Caldas é de $1.745 \mathrm{~mm}$, sendo a pluviosidade mínima registrada no mês de julho, com índices próximos a 25 mm, e pluviosidades máximas registradas em janeiro, com valores da ordem de $297 \mathrm{~mm}$ (FERNANDES et al., 2003). A população total do município é de 152.435 (IBGE, 2010), entretanto, a população de influência na bacia em estudo é de 130.385 habitantes (DMAE, 2008).

Para a quantificação da carga de origem doméstica foram levantadas informações sobre a população real influente dentro dos limites da bacia. Desta forma, foi calculada a carga potencial, utilizando o valor per capita de 0,054 kgDBO/dia.habitante. Segundo Mota (1997) esta carga per capita é bastante utilizada na literatura. A Equação 01 descreve os cálculos realizados

$$
C P=D B O_{\text {esgoto }} \times Q_{\text {esgoto }} \times P O P_{\text {bacia }}
$$

Onde:

$\mathrm{CP}=$ Carga Potencial do esgoto doméstico $\left(\mathrm{kgDBO} \cdot \mathrm{dia}^{-1}\right)$

$\mathrm{DBO}_{\text {esgoto }}=300 \mathrm{mg} \cdot \mathrm{L}^{-1}$

$\mathrm{Q}_{\text {esgoto }}=180$ L.(dia.habitante) $)^{-1}$

$\mathrm{POP}_{\text {bacia }}=$ População de influência na bacia (habitantes)

Os principais dados relacionados a este item foram encontrados no DEPARTAMENTO MUNICIPAL DE ÁGUA E ESGOTO (DMAE). Não há Estação de Tratamento de Esgoto (ETE) em operação na área de influência da bacia.

O estabelecimento dos pontos de coleta teve como base a área de influência das sub-bacias e a urbanização existente. Desta forma, foram determinados 06 (seis) sítios de coleta. A localidade dos pontos de coleta assim como a rede de drenagem da bacia em estudo estão representados na Figura 2.

Os fatores de qualidade da água foram medidas em campo e em laboratório. A Tabela 1 apresenta um resumo dos fatores e os métodos empregados no estudo.
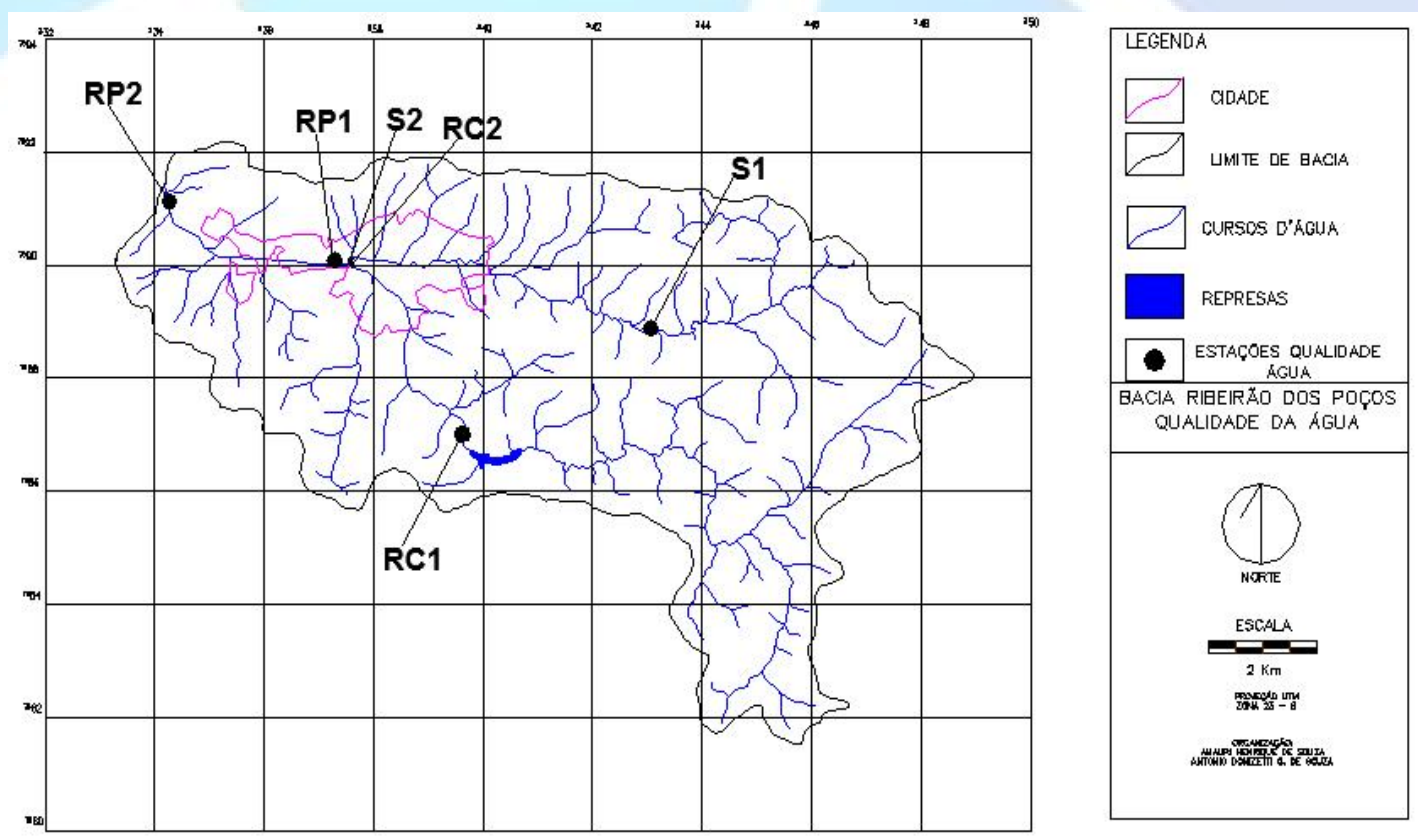

Figura 2. Sítios de amostragem da qualidade da água e área urbana da bacia do Ribeirão dos Poços. 
Tabela 1 - Variáveis físicas e químicas medidas na bacia do Ribeirão dos Poços.

\begin{tabular}{|c|c|c|}
\hline Variáveis & Método de Análise & Unidades \\
\hline Temperatura do ar & Termômetro & ${ }^{\circ} \mathrm{C}$ \\
\hline Temperatura da água & \multirow{4}{*}{$\begin{array}{l}\text { Leitura direta em campo } \\
\text { Eutech Instruments (modelo PCD 650) }\end{array}$} & ${ }^{\circ} \mathrm{C}$ \\
\hline $\mathrm{pH}$ & & - \\
\hline Condutividade & & $\mu \mathrm{S} . \mathrm{cm}^{-1}$ \\
\hline Oxigênio Dissolvido & & mg. $\mathrm{L}^{-1}$ \\
\hline Saturação OD & Cálculos (Golterman et al., 1978) & $\%$ \\
\hline Turbidez & $\begin{array}{c}\text { Turbidímetro de bancada } \\
\text { Vernier (modelo TRB-BTA) }\end{array}$ & NTU \\
\hline Alcalinidade & Titulação $\mathrm{H}_{2} \mathrm{SO}_{4}(\mathrm{APHA}, 2005)$ & mg. $L^{-1}$ \\
\hline $\begin{array}{ll}\text { Sólidos } & \text { Suspensos } \\
\text { Totais (STS) } & \end{array}$ & Gravimetria (APHA, 2005) & $\mathrm{mg} \cdot \mathrm{L}^{-1}$ \\
\hline
\end{tabular}

No sítio RP2, por ser localizado na foz da bacia (exutório) foram feitas medidas de vazão $\left(\mathrm{m}^{3} \cdot \mathrm{s}^{-1}\right)$. Para a determinação da vazão $\left(\mathrm{m}^{3} \cdot \mathrm{s}^{-1}\right)$ foi utilizado o método flutuador. $\mathrm{O}$ método consiste em determinar a área da seção do rio e a velocidade média do fluxo de água que passa nesta seção. A área é determinada por meio da medição da largura do rio e da profundidade em um número significativo de pontos ao longo da seção transversal do rio, denominados de verticais.

O sítio RP2 também foi selecionado para cálculos da carga de sólidos suspensos expressa em kg.dia ${ }^{-1}$, devido a este local representar toda a contribuição final da bacia. Os dados da qualidade da água foram analisados segundo a variação espacial e temporal encontrada e interrelacionada ao uso e ocupação do solo existente na bacia. Para a variação temporal os dados foram divididos em dois períodos sazonais, sendo o período seco (abril a setembro) e o período chuvoso (outubro a março).

Os resultados foram analisado estatisticamente com a construção de matrizes de correlação utilizando a correlação de Spearman e emprego da técnica de Análise de Agrupamento (Cluster Analysis). Esta última permitiu uma análise comparativa e uma ordenação das estações de coleta segundo as características próprias e interferências existentes.

Para a verificação se houve diferenças entre os períodos de sazonais (inverno e verão) e entre as estações de coleta, foi aplicado o teste não paramétrico de Kruskal-Wallis (teste H). Este teste é muito utilizado em avaliações da qualidade da água, pois os dados geralmente não apresentam distribuição normal.

\section{RESULTADOS E DISCUSSÃO}

Com uma população estimada em 130.385 habitantes na bacia estudada, foi obtida uma carga potencial doméstica de 7.040,79 kg.DBO.dia ${ }^{-1}$. Os dados obtidos para vazão nos períodos de inverno e verão foram respectivamente: $2,21 \mathrm{~m}^{3} \cdot \mathrm{s}^{-1}$ e $7,24 \mathrm{~m}^{3} \cdot \mathrm{s}^{-1}$. A carga de STS foi de 1.368,86 kg.dia ${ }^{-1}$ no inverno e $2.8614,63 \mathrm{~kg}_{\text {dia }}{ }^{-1}$ para as coletas realizadas no verão. A Figura 3 representa a pluviometria média, máxima e mínima dos meses entre o período de 2002 a 2011. 


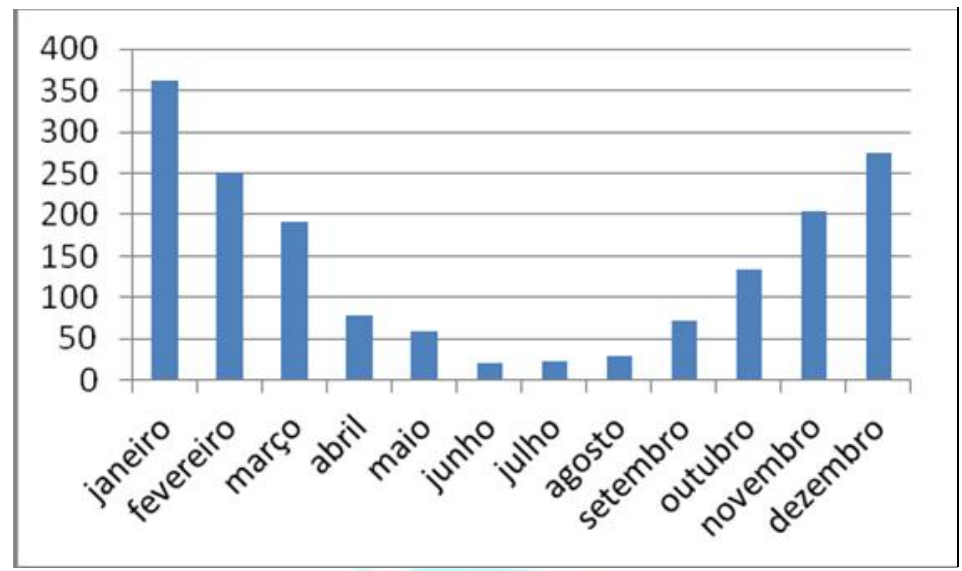

Figura 3. Pluviometria média (mm) da bacia Ribeirão de Poços no período 2002-2011. Fonte: Cooperativa Regional dos Cafeicultores de Poços de Caldas

Os resultados da qualidade da água para os dois períodos (inverno e verão) estão apresentados nas Tabelas 2 e 3, onde também está representada uma estatística descritiva dos dados.

Os resultados obtidos nos dois períodos sazonais indicaram um gradiente de aumento nos fatores no sentido montante-jusante, especialmente entre as duas primeiras estações dos rios Ribeirão de Caldas (RC1 e RC2) e Ribeirão da Serra (S1 e S2), as quais podem estar relacionados aos impactos da área urbana sobre os corpos d'águas,

A condutividade sofreu grande aumento após o curso d'água atravessar a zona urbana do município, variando de seu valor mínimo de 40,36 S.cm ${ }^{-1}$ em S1 ao valor máximo de 144,4 S.cm ${ }^{-1}$ em S2. Os fatores sólidos totais suspensos, sólidos totais dissolvidos e alcalinidade também apresentaram o mesmo padrão de variação refletindo provavelmente a influência dos despejos de fontes pontuais e difusas de poluição sobre o curso.

$\mathrm{O}$ oxigênio dissolvido registrou um valor mínimo de $2,71 \mathrm{mg} . \mathrm{L}^{-1}$ no ponto próximo ao exutório (ponto de maior vazão) da bacia estudada (RC2).

Os sítios de coleta localizados no Ribeirão de Caldas apresentaram altos valores de turbidez, o que deve estar relacionado ao fato do curso d'água passar por uma área rural da cidade. $\mathrm{O}$ pH não apresentou um claro padrão de aumento e se manteve constante durante o curso dos rios com valores de aproximadamente 6,00 .

Os resultados obtidos nas análises de água demonstraram uma significativa influência da área urbana sobre os corpos hídricos. Os pontos RC1 e S1 situados mais a montante da área urbana, nos rios Ribeirão de Caldas e Ribeirão da Serra, respectivamente, demonstraram os melhores resultados dos fatores estudas em ambas as estações (inverno e verão), devido provavelmente a sua localização mais distante da área urbanizada.

O ponto S2 demonstrou ser a mais impactada dentre os escolhidos, refletindo sua localização a jusante de uma área densamente urbanizada, local com baixa vazão e muitas fontes pontuais e difusas. Os três pontos restantes (RC2, RP1 e RP2) apresentaram comportamento muito semelhantes com relação à qualidade da água em praticamente todas os fatores, com destaque para os fatores turbidez, alcalinidade e condutividade. A Tabela 4 representa a matriz de correlação $(r$ de Sperman) para os fatores analisadas, estações e períodos, foram consideradas significativas as correlações com $\mathrm{p}<0,05$.

As análises de agrupamento estão representadas nos dendogramas de similaridade espacial das estações de coleta para cada período sazonal (Figura 4). No inverno/2012 observou-se a formação de dois grupos distintos. O primeiro grupo formado pelas estações $\mathrm{S} 1$ e RC1 e o segundo grupo formado pelos sítios RC2, RP1 e RP2. Esta distribuição dos sítios reflete as influências e impactos da área urbana sobre a qualidade da água. 
O primeiro grupo formado ( $\mathrm{S} 1$ e $\mathrm{RC} 1$ ) apresenta níveis de qualidade da água superior, principalmente relacionada ao oxigênio dissolvido e condutividade. O sítio RC1 encontra-se montante da área urbana, jusante à barragem Saturnino de Brito e o S1 a montante de área densamente urbanizada do município. Já o segundo grupo de estações, situa-se totalmente na área urbanizada, neste caso com baixos valores de oxigênio. O ponto S2 mostrou-se um pouco isolado das demais, o que pode estar relacionado aos altos valores de condutividade, sólidos totais dissolvidos e alcalinidade, médias de 136,60 $\mathrm{S} . \mathrm{cm}^{-1}, \quad 53,78 \mathrm{mg} . \mathrm{L}^{-1}$ e $41,33 \mathrm{mg} . \mathrm{L}^{-1}$ respectivamente, as maiores entre as médias dos sítios. A estação se localiza a jusante de uma área densamente urbanizada, próxima ao encontro com o Ribeirão de Caldas, local com baixa vazão e muitas fontes pontuais e difusas.

Tabela 2 - Resultados obtidos dos fatores físicas e químicas da água para o período de inverno.

\begin{tabular}{|c|c|c|c|c|c|c|c|c|c|c|c|c|}
\hline Sítios & $\begin{array}{l}\text { Estatística } \\
\text { Descritiva }\end{array}$ & $\begin{array}{l}\text { Temp. } \\
\left({ }^{\circ} \mathrm{C}\right)\end{array}$ & $\begin{array}{l}\text { Cond. } \\
(\text { S/cm) }\end{array}$ & $\begin{array}{c}\text { STD } \\
\left(\mathrm{mg.L}^{-1}\right)\end{array}$ & $\begin{array}{c}\text { OD } \\
(\mathrm{mg} / \mathrm{L})\end{array}$ & $\begin{array}{l}\text { Turb. } \\
\text { (NTU) }\end{array}$ & pH & $\begin{array}{c}\text { Sat.OD } \\
(\%)\end{array}$ & $\begin{array}{c}\text { Alcal. } \\
\left(\mathrm{mg}^{-L^{-1}}\right)\end{array}$ & $\begin{array}{c}\text { STS } \\
\left(\mathrm{mg}^{-L^{-1}}\right)\end{array}$ & $\begin{array}{c}\text { STS } \\
\text { Org. } \\
\left(\mathrm{mg.L}^{-1}\right)\end{array}$ & $\begin{array}{c}\text { STS } \\
\text { Inorg. } \\
\left(\text { mg.L }^{-1}\right)\end{array}$ \\
\hline \multirow[t]{6}{*}{ S1 } & $\mathrm{N}$ & 6,00 & 6,00 & 6,00 & 6,00 & 6,00 & 6,00 & 6,00 & 6,00 & 6,00 & 6,00 & 6,00 \\
\hline & Mínimo & 13,60 & 40,36 & 14,09 & 6,32 & 6,30 & 5,78 & 77,01 & 9,00 & 2,00 & 0,80 & 1,20 \\
\hline & Máximo & 16,50 & 65,53 & 24,16 & 7,90 & 10,47 & 6,20 & 92,63 & 11,00 & 7,00 & 3,82 & 4,40 \\
\hline & Média & 15,35 & 53,56 & 18,68 & 7,26 & 8,39 & 6,05 & 86,79 & 10,00 & 4,72 & 2,30 & 1,73 \\
\hline & Mediana & 15,80 & 55,64 & 18,84 & 7,30 & 8,45 & 6,11 & 86,48 & 10,00 & 5,18 & 2,30 & 1,73 \\
\hline & Desv.Pad. & 1,09 & 8,69 & 3,58 & 0,53 & 1,75 & 0,16 & 5,19 & 0,58 & 1,62 & 1,06 & 1,22 \\
\hline \multirow[t]{6}{*}{ S2 } & $\mathrm{N}$ & 6,00 & 6,00 & 6,00 & 6,00 & 6,00 & 6,00 & 6,00 & 6,00 & 6,00 & 6,00 & 6,00 \\
\hline & Mínimo & 16,80 & 129,80 & 49,88 & 3,51 & 18,90 & 5,42 & 43,73 & 38,00 & 23,45 & 16,90 & 6,55 \\
\hline & Máximo & 18,20 & 144,40 & 58,05 & 5,77 & 53,80 & 6,27 & 73,15 & 45,00 & 66,00 & 37,50 & 28,50 \\
\hline & Média & 17,70 & 136,60 & 53,78 & 4,71 & 35,64 & 5,98 & 59,23 & 41,50 & 41,33 & 24,38 & 16,44 \\
\hline & Mediana & 17,95 & 135,20 & 53,80 & 4,84 & 36,25 & 6,00 & 61,09 & 41,50 & 41,33 & 24,38 & 16,44 \\
\hline & Desv.Pad. & 0,49 & 4,69 & 2,73 & 0,79 & 11,81 & 0,28 & 10,39 & 2,22 & 13,84 & 7,34 & 6,87 \\
\hline \multirow[t]{6}{*}{ RC1 } & $\mathrm{N}$ & 6,00 & 6,00 & 6,00 & 6,00 & 6,00 & 6,00 & 6,00 & 6,00 & 6,00 & 6,00 & 6,00 \\
\hline & Mínimo & 16,20 & 24,31 & 5,06 & 4,25 & 14,80 & 5,56 & 53,59 & 11,00 & 5,78 & 2,29 & 3,11 \\
\hline & Máximo & 18,80 & 25,61 & 10,38 & 7,88 & 23,73 & 6,30 & 98,11 & 13,00 & 10,75 & 6,75 & 4,50 \\
\hline & Média & 18,07 & 24,81 & 9,30 & 6,85 & 18,29 & 5,73 & 86,56 & 12,33 & 7,31 & 3,30 & 4,01 \\
\hline & Mediana & 18,45 & 24,49 & 10,06 & 7,24 & 17,43 & 5,62 & 92,19 & 12,50 & 6,79 & 2,63 & 4,14 \\
\hline & Desv.Pad. & 0,88 & 0,54 & 1,91 & 1,21 & 3,06 & 0,26 & 15,16 & 0,75 & 1,63 & 1,56 & 0,47 \\
\hline \multirow[t]{6}{*}{ RC2 } & $\mathrm{N}$ & 6,00 & 6,00 & 6,00 & 6,00 & 6,00 & 6,00 & 6,00 & 6,00 & 6,00 & 6,00 & 6,00 \\
\hline & Mínimo & 17,50 & 79,49 & 30,87 & 3,72 & 14,17 & 5,33 & 46,50 & 25,00 & 7,00 & 4,44 & 2,25 \\
\hline & Máximo & 18,60 & 89,79 & 36,83 & 6,73 & 50,60 & 6,22 & 85,98 & 33,00 & 45,20 & 17,60 & 27,60 \\
\hline & Média & 18,20 & 84,24 & 33,81 & 5,28 & 23,60 & 5,87 & 67,09 & 28,33 & 19,95 & 9,23 & 10,73 \\
\hline & Mediana & 18,50 & 83,91 & 33,28 & 5,40 & 14,65 & 5,92 & 68,79 & 28,00 & 10,21 & 6,54 & 3,67 \\
\hline & Desv.Pad. & 0,50 & 3,82 & 1,88 & 1,27 & 13,85 & 0,28 & 16,61 & 2,49 & 16,14 & 5,27 & 10,89 \\
\hline \multirow[t]{6}{*}{ RP1 } & $\mathrm{N}$ & 6,00 & 6,00 & 6,00 & 5,00 & 6,00 & 6,00 & 6,00 & 6,00 & 6,00 & 6,00 & 6,00 \\
\hline & Mínimo & 17,20 & 102,90 & 39,29 & 3,18 & 18,73 & 5,75 & 39,54 & 26,00 & 14,47 & 9,21 & 5,26 \\
\hline & Máximo & 18,50 & 112,70 & 45,57 & 5,59 & 28,27 & 6,31 & 99,81 & 35,00 & 37,00 & 20,33 & 16,67 \\
\hline & Média & 18,02 & 108,28 & 43,16 & 4,26 & 25,41 & 6,02 & 61,45 & 32,50 & 25,32 & 14,46 & 10,85 \\
\hline & Mediana & 18,25 & 108,75 & 43,96 & 4,50 & 27,07 & 5,98 & 57,11 & 33,50 & 25,54 & 14,14 & 10,86 \\
\hline & Desv.Pad. & 0,49 & 2,90 & 2,09 & 0,85 & 3,44 & 0,18 & 19,95 & 3,10 & 7,25 & 3,44 & 4,05 \\
\hline \multirow[t]{6}{*}{ RP2 } & $\mathrm{N}$ & 6,00 & 6,00 & 6,00 & 6,00 & 6,00 & 6,00 & 6,00 & 6,00 & 6,00 & 6,00 & 6,00 \\
\hline & Mínimo & 17,00 & 85,46 & 32,39 & 2,71 & 2,50 & 5,65 & 34,47 & 27,00 & 5,00 & 2,00 & 1,71 \\
\hline & Máximo & 18,40 & 112,70 & 36,87 & 5,55 & 18,20 & 6,34 & 70,36 & 31,00 & 10,30 & 7,27 & 2,33 \\
\hline & Média & 17,98 & 93,80 & 35,68 & 3,46 & 11,33 & 5,94 & 43,70 & 29,50 & 7,00 & 4,42 & 2,58 \\
\hline & Mediana & 18,20 & 91,05 & 36,08 & 3,14 & 11,25 & 5,93 & 39,63 & 29,50 & 6,86 & 4,62 & 2,67 \\
\hline & Desv.Pad. & 0,50 & 8,77 & 1,51 & 0,96 & 4,71 & 0,25 & 12,29 & 1,38 & 1,63 & 1,69 & 0,52 \\
\hline
\end{tabular}


Tabela 3 - Resultados obtidos dos fatores físicas e químicas da água para o período de verão.

\begin{tabular}{|c|c|c|c|c|c|c|c|c|c|c|c|c|}
\hline Sítios & $\begin{array}{l}\text { Estatística } \\
\text { Descritiva }\end{array}$ & $\begin{array}{l}\text { Temp. } \\
\left({ }^{\circ} \mathrm{C}\right)\end{array}$ & $\begin{array}{l}\text { Cond. } \\
(\mathrm{S} / \mathrm{cm})\end{array}$ & $\begin{array}{c}\text { STD } \\
\left(\mathrm{mg.L} \mathbf{L}^{-1}\right)\end{array}$ & $\begin{array}{c}\text { OD } \\
(\mathrm{mg} / \mathrm{L})\end{array}$ & pH & $\begin{array}{l}\text { Turb. } \\
\text { (NTU) }\end{array}$ & $\begin{array}{c}\text { Sat.OD } \\
(\%)\end{array}$ & $\begin{array}{c}\text { Alcal. } \\
\left(\mathrm{mg.L}^{-1}\right)\end{array}$ & $\begin{array}{c}\text { STS } \\
\left(\mathrm{mg.L}^{-1}\right)\end{array}$ & $\begin{array}{c}\text { STS } \\
\text { Org. } \\
\left(\mathrm{mg.L}^{-1}\right)\end{array}$ & $\begin{array}{c}\text { STS } \\
\text { Inorg. } \\
\left(\text { mg.L }^{-1}\right)\end{array}$ \\
\hline \multirow[t]{6}{*}{ S1 } & $\mathrm{N}$ & 6,00 & 6,00 & 6,00 & 4,00 & 5,00 & 6,00 & 4,00 & 4,00 & 6,00 & 6,00 & 6,00 \\
\hline & Mínimo & 21,50 & 23,77 & 11,78 & 6,60 & 5,90 & 22,70 & 89,54 & 21,00 & 6,75 & 2,75 & 4,00 \\
\hline & Máximo & 22,60 & 38,17 & 18,42 & 7,10 & 6,17 & 92,00 & 97,35 & 25,50 & 31,73 & 9,60 & 22,13 \\
\hline & Média & 22,08 & 33,33 & 16,50 & 6,90 & 6,03 & 50,63 & 94,32 & 23,50 & 17,92 & 5,63 & 12,29 \\
\hline & Mediana & 22,05 & 35,64 & 17,00 & 6,95 & 6,05 & 42,40 & 95,19 & 23,75 & 14,36 & 4,65 & 9,77 \\
\hline & Desv.Pad. & 0,39 & 5,15 & 2,22 & 0,19 & 0,10 & 26,51 & 2,98 & 1,84 & 9,72 & 2,53 & 7,21 \\
\hline \multirow[t]{6}{*}{ S2 } & $\mathrm{N}$ & 6,00 & 5,00 & 5,00 & 5,00 & 6,00 & 6,00 & 5,00 & 5,00 & 6,00 & 6,00 & 6,00 \\
\hline & Mínimo & 22,90 & 79,03 & 40,11 & 6,00 & 5,38 & 23,50 & 84,57 & 56,50 & 7,73 & 5,45 & 2,27 \\
\hline & Máximo & 24,20 & 83,17 & 93,56 & 7,90 & 6,70 & 84,40 & 110,01 & 66,00 & 51,64 & 15,64 & 36,00 \\
\hline & Média & 23,60 & 81,71 & 51,85 & 6,92 & 6,20 & 41,33 & 96,48 & 61,50 & 27,41 & 11,11 & 16,30 \\
\hline & Mediana & 23,50 & 82,71 & 41,89 & 6,90 & 6,36 & 30,65 & 95,29 & 61,00 & 24,11 & 11,45 & 14,20 \\
\hline & Desv.Pad. & 0,44 & 1,56 & 20,87 & 0,61 & 0,42 & 20,76 & 8,30 & 3,18 & 14,59 & 3,55 & 11,92 \\
\hline \multirow[t]{6}{*}{ RC1 } & $\mathrm{N}$ & 6,00 & 5,00 & 5,00 & 5,00 & 6,00 & 5,00 & 5,00 & 6,00 & 6,00 & 6,00 & 6,00 \\
\hline & Mínimo & 22,60 & 20,03 & 10,03 & 6,20 & 5,19 & 168,80 & 86,65 & 24,25 & 20,00 & 7,14 & 5,00 \\
\hline & Máximo & 26,00 & 21,89 & 12,54 & 9,40 & 6,44 & 280,50 & 133,46 & 30,00 & 67,69 & 19,33 & 50,00 \\
\hline & Média & 23,78 & 21,09 & 11,09 & 7,40 & 5,97 & 203,46 & 104,12 & 27,08 & 46,31 & 14,28 & 32,04 \\
\hline & Mediana & 23,45 & 21,23 & 11,01 & 7,00 & 6,04 & 192,20 & 97,36 & 27,13 & 53,54 & 14,63 & 40,85 \\
\hline & Desv.Pad. & 1,20 & 0,70 & 0,87 & 1,11 & 0,44 & 40,10 & 15,89 & 2,29 & 18,87 & 4,02 & 16,80 \\
\hline \multirow[t]{6}{*}{ RC2 } & $\mathrm{N}$ & 6,00 & 6,00 & 6,00 & 5,00 & 6,00 & 5,00 & 5,00 & 5,00 & 6,00 & 6,00 & 6,00 \\
\hline & Mínimo & 23,50 & 42,36 & 21,68 & 6,50 & 5,25 & 118,10 & 93,41 & 36,50 & 11,67 & 6,25 & 5,42 \\
\hline & Máximo & 25,70 & 51,66 & 28,81 & 8,10 & 6,55 & 208,30 & 115,29 & 45,75 & 64,74 & 16,00 & 48,95 \\
\hline & Média & 24,18 & 46,31 & 24,48 & 7,30 & 6,23 & 156,12 & 103,08 & 41,25 & 46,83 & 12,78 & 34,06 \\
\hline & Mediana & 23,85 & 44,81 & 23,39 & 7,50 & 6,43 & 152,20 & 105,33 & 41,00 & 51,28 & 13,13 & 36,89 \\
\hline & Desv.Pad. & 0,79 & 3,73 & 2,50 & 0,61 & 0,45 & 29,99 & 8,34 & 3,19 & 17,06 & 3,23 & 14,28 \\
\hline \multirow[t]{6}{*}{ RP1 } & $\mathrm{N}$ & 6,00 & 6,00 & 6,00 & 5,00 & 6,00 & 5,00 & 5,00 & 5,00 & 6,00 & 6,00 & 6,00 \\
\hline & Mínimo & 23,40 & 53,06 & 27,22 & 6,30 & 4,96 & 87,70 & 86,38 & 41,50 & 14,58 & 7,50 & 7,08 \\
\hline & Máximo & 25,20 & 60,95 & 33,66 & 8,30 & 6,63 & 177,80 & 115,72 & 50,50 & 60,45 & 16,11 & 45,91 \\
\hline & Média & 23,92 & 56,85 & 29,78 & 7,06 & 6,14 & 131,28 & 98,99 & 46,90 & 41,06 & 12,79 & 28,28 \\
\hline & Mediana & 23,50 & 56,49 & 29,74 & 6,70 & 6,37 & 133,30 & 93,30 & 49,50 & 42,28 & 13,27 & 29,00 \\
\hline & Desv.Pad. & 0,69 & 2,88 & 2,16 & 0,74 & 0,56 & 30,84 & 10,57 & 3,67 & 14,82 & 3,11 & 12,12 \\
\hline \multirow[t]{6}{*}{ RP2 } & $\mathrm{N}$ & 6,00 & 6,00 & 6,00 & 5,00 & 6,00 & 5,00 & 5,00 & 5,00 & 6,00 & 6,00 & 6,00 \\
\hline & Mínimo & 23,30 & 59,84 & 30,68 & 5,00 & 5,84 & 81,40 & 70,91 & 52,50 & 16,96 & 8,70 & 8,26 \\
\hline & Máximo & 25,90 & 68,33 & 38,66 & 6,60 & 6,65 & 164,30 & 93,25 & 59,50 & 69,00 & 20,00 & 51,00 \\
\hline & Média & 24,37 & 64,70 & 34,40 & 6,02 & 6,29 & 124,50 & 83,12 & 55,20 & 45,70 & 13,88 & 31,82 \\
\hline & Mediana & 24,30 & 65,16 & 34,36 & 6,20 & 6,28 & 122,30 & 87,60 & 55,00 & 43,55 & 12,69 & 31,13 \\
\hline & Desv.Pad. & 0,87 & 2,75 & 2,43 & 0,56 & 0,29 & 27,21 & 9,50 & 2,64 & 17,38 & 3,94 & 13,70 \\
\hline
\end{tabular}

Tabela 4 - Matriz de correlação entre os fatores medidas. Correlação $r$ de Spearman. Em destaque as correlações não significativas a $\mathrm{p}<0,05$.

\begin{tabular}{|c|c|c|c|c|c|c|c|c|c|c|c|}
\hline & Temperatura & Condutividade & STD & OD & pH & Turbidez & $\%$ OD & Alcalinidade & STS & STS (org) & STS (ing) \\
\hline Temperatura & 1 & $-0,368$ & $-0,109$ & 0,273 & $-0,665$ & 0,754 & $\mathbf{0 , 5 7 0}$ & 0,576 & $-0,721$ & $-0,710$ & $-0,737$ \\
\hline Condutividade & & 1 & 0,942 & $-0,701$ & $\mathbf{0 , 5 3 2}$ & $-0,394$ & $-0,667$ & 0,408 & 0,566 & 0,612 & 0,509 \\
\hline STD & & & 1 & $-0,594$ & $\mathbf{0 , 3 6 0}$ & $-0,178$ & $-0,488$ & 0,625 & 0,354 & 0,405 & 0,296 \\
\hline OD & & & & 1 & $-0,423$ & 0,441 & 0,848 & $-0,310$ & $-0,454$ & $-0,502$ & $\begin{array}{l}-0,398 \\
\end{array}$ \\
\hline pH & & & & & 1 & $-0,663$ & $-0,538$ & $-0,130$ & $\mathbf{0 , 8 3 7}$ & 0,816 & 0,845 \\
\hline Turbidez & & & & & & $\underline{1}$ & 0,618 & 0,382 & $-0,664$ & $-0,653$ & $-0,679$ \\
\hline$\%$ OD & & & & & & & 1 & $-0,021$ & $-0,594$ & $-0,641$ & $-0,550$ \\
\hline Alcalinidade & & & & & & & & 1 & $-0,244$ & $-0,215$ & $-0,288$ \\
\hline STS & & & & & & & & & 1 & 0,976 & 0,982 \\
\hline STS(org) & & & & & & & & & & 1 & $\mathbf{0 , 9 3 7}$ \\
\hline STS(ing) & & & & & & & & & & & 1 \\
\hline
\end{tabular}



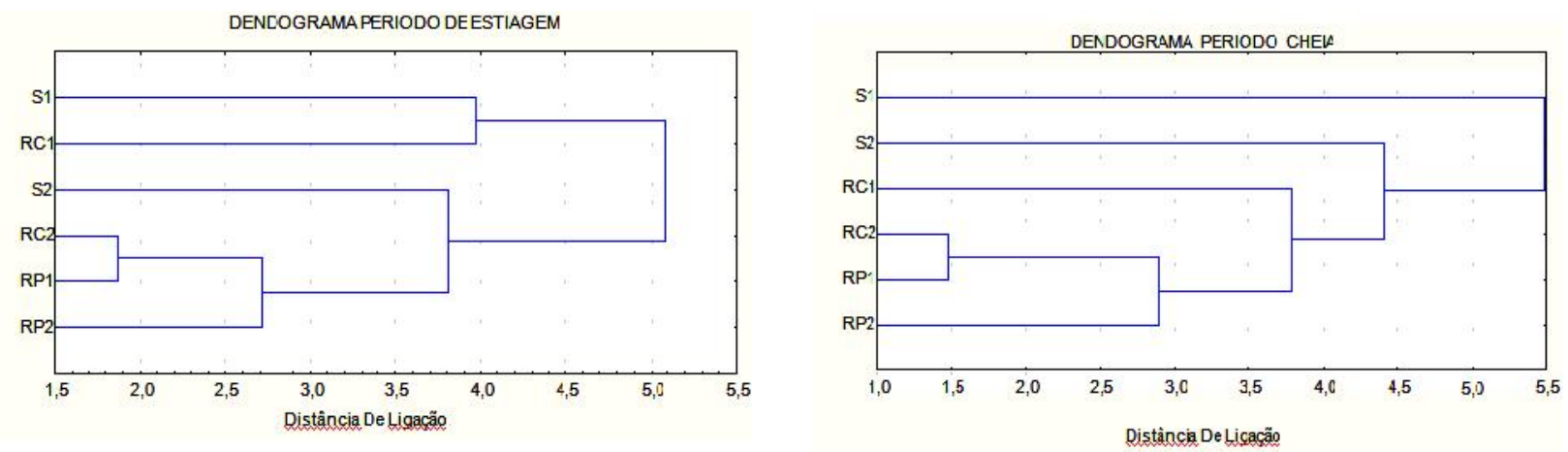

Figura 4. Dendogramas de similaridade espacial entre as estações de coleta para os valores medianos da qualidade da água no inverno (estiagem) e verão (cheia)

No verão/2013 observou-se a formação de um grupo com os sítios RC2, RP1 e RP2, enquanto, os três sítios restantes ficaram isolados. Os três pontos agrupados se encontram a jusante de uma área urbanizada, o ponto RC2 se encontra próximo ao exutório da sub-bacia do Ribeirão de Caldas, pouco antes de seu encontro com o Ribeirão da Serra. Os sítios RP1 e RP2 se localizam após este encontro no curso d'água principal da bacia, denominado Ribeirão de Poços. Os três pontos possuem grande semelhança em todas os fatores, com destaque para os fatores turbidez, alcalinidade e condutividade. A estação RC1 se encontra afastada das outras devido a melhores índices de qualidade da água, com exceção para a variável turbidez, que devido à área rural pela qual o corpo d'água atravessa, atinge valores elevados no período de cheia.

No rio Ribeirão da Serra, a estação S2 se destaca por ser a mais impactada entre todas as estações em relação às variáveis condutividade, alcalinidade e sólidos totais dissolvidos, assim como foi registrado no período de estiagem. O outro sítio S1 deste corpo hídrico destacou-se por apresentar níveis de qualidade da água superior em quase todas os fatores estudadas. Este sítio se encontra o mais afastado da área urbanizada entre todos os outros, o que indica prováveis impactos antrópicos da área urbana sobre a qualidade da água da bacia.

Pode-se observar o afastamento do ponto $\mathrm{RC} 1 \mathrm{com}$ o ponto $\mathrm{S} 1$ no período de estiagem para o período de cheia, o impacto causado pelo aumento das chuvas é significativamente maior neste ponto, causando um grande aumento na variável turbidez, atingindo a maior média entre todos os sítios.

O resultado do teste não paramétrico de Kuskal-Wallis (teste $\mathrm{H}$ ) está representado na Tabela 5. Fixando-se os períodos sazonais como fonte de variação houve a diferenciação de todas os fatores de qualidade da água considerada com valores de $\mathrm{H}$ significativos $(\mathrm{p}<1 \%)$, exceto a variável sólidos totais dissolvidos. Para as estações de coletas a temperatura, $\mathrm{pH}$, turbidez, porcentagem de oxigênio dissolvido e sólidos totais dissolvidos (orgânicos e inorgânicos) não apresentaram diferenciação significativa a $\mathrm{p}<0,01$.

Nos resultados do teste não paramétrico de Kuskal-Wallis (teste $\mathrm{H}$ ), quando foram fixados os períodos sazonais como fonte de variação, houve a diferenciação de todas os fatores de qualidade da água considerada com valores de $\mathrm{H}$ significativos $(\mathrm{p}<1 \%)$, exceto a variável sólidos totais dissolvidos. Este teste também foi realizado por Alves et al. (2008) para verificar diferença significativa entre os pontos amostrados em período sazonais com resultados satisfatórios e semelhantes.

O período de maior pluviometria apresentou valores baixos para os fatores OD, sólidos totais suspensos, alcalinidade, condutividade e turbidez. Durante as estações chuvosas, há um aumento da densidade de bactérias nas águas, tendo em vista o maior carreamento de material fecal por meio do escoamento superficial em áreas rurais e urbanas, além de galerias pluviais e córregos, comprometendo, dessa maneira, a qualidade da água (WHO, 2003). A turbidez e sólidos totais 
suspensos sofreram grande variação entre os períodos, atingindo altos valores no período chuvoso, o que caracteriza a ação exercida pelo arraste superficial (TAVARES, 2005).

Tabela 5 - Resultados encontrados no teste Kruskal-Wallis (teste $\mathrm{H}$ ) para variáveis registradas durante o período 2012-2013. Em negrito, valores não significativos a $p<0,01(*)$.

\begin{tabular}{|c|c|c|}
\hline \multirow[t]{2}{*}{ Variáveis } & \multicolumn{2}{|c|}{ Fontes de Variação } \\
\hline & $\begin{array}{c}\text { Espacial } \\
\text { Sítios de coleta } \\
\text { (Entre os } 06 \text { sítios) }\end{array}$ & $\begin{array}{c}\text { Temporal } \\
\text { Períodos } \\
\text { (Entre a estiagem e chuva) }\end{array}$ \\
\hline TEMP & $\begin{array}{c}\mathbf{8 , 1 5 5} \\
\mathrm{p}=0,148\end{array}$ & $\begin{array}{c}53,336 \\
\mathrm{p}=0,000\end{array}$ \\
\hline COND & $\begin{array}{c}48,208 \\
P=0,000\end{array}$ & $\begin{array}{c}16,057 \\
p=0,000\end{array}$ \\
\hline STD & $\begin{array}{c}58,966 \\
p=0,000\end{array}$ & $\begin{array}{c}\mathbf{3 , 2 7 5} \\
\mathrm{p}=0,070\end{array}$ \\
\hline OD & $\begin{array}{c}21,665 \\
p=0,001\end{array}$ & $\begin{array}{c}11,374 \\
\mathrm{p}=0,001\end{array}$ \\
\hline pH & $\begin{array}{c}\mathbf{6 , 3 5 6} \\
p=0,273\end{array}$ & $\begin{array}{c}43,895 \\
p=0,000\end{array}$ \\
\hline TURB & $\begin{array}{c}\mathbf{0 , 5 1 5} \\
\mathrm{p}=0,992\end{array}$ & $\begin{array}{c}50,093 \\
p=0,000\end{array}$ \\
\hline$\%$ OD & $\begin{array}{c}\mathbf{1 3 , 1 2 9} \\
\mathrm{p}=0,022\end{array}$ & $\begin{array}{c}27,865 \\
p=0,000\end{array}$ \\
\hline ALCAL & $\begin{array}{c}45,249 \\
p=0,000\end{array}$ & $\begin{array}{c}15,643 \\
p=0,000\end{array}$ \\
\hline STS & $\begin{array}{c}\mathbf{5 , 6 3 1} \\
p=0,344\end{array}$ & $\begin{array}{c}53,271 \\
p=0,000\end{array}$ \\
\hline STS $_{(\text {ORG })}$ & $\begin{array}{c}\mathbf{6 , 3 4 4} \\
p=0,274\end{array}$ & $\begin{array}{c}53,265 \\
p=0,000\end{array}$ \\
\hline STS $_{\text {(INORG) }}$ & $\begin{array}{c}\mathbf{4 , 1 0 6} \\
\mathrm{p}=0,534\end{array}$ & $\begin{array}{c}53,263 \\
p=0,000\end{array}$ \\
\hline
\end{tabular}

(*) Os valores em negrito indicam que não há diferenças significativas para os fatores de qualidade da água a probabilidade de $\mathrm{p}<0,01$.

As análises estatísticas exploratórias utilizadas, análise de agrupamento(kg/dia) e KuskalWallis (teste $\mathrm{H}$ ), apresentaram uma classificação dos fatores da qualidade da água no aspecto espacial como sazonal. As técnicas multivariadas forneceram um auxilio a interpretação dos dados produzidos e em programas de monitoramento mais amplos, com maior numero de variáveis, podem ser uteis na transformação dos dados em informações.

Em síntese, a bacia estudada apresentou a melhor qualidade nas estações mais a montante da área urbana e qualidade inferior em estações localizadas em áreas densamente urbanizadas, com poucas variações entre os períodos sazonais. Estes resultados reforçam a hipótese proposta no trabalho de que a área urbanizada altera a qualidade da água na bacia hidrográfica do Ribeirão dos Poços (Poços de Caldas-MG). 


\section{CONCLUSÕES}

A avaliação da qualidade da água realizada na bacia do Ribeirão dos Poços demonstrou a influência da área urbana sobre a qualidade da água relacionada provavelmente a lançamentos pontuais e difusos. A estação S1, localizada a jusante de uma área densamente urbanizada, se mostrou adequada como sítio de referência para futura rede de monitoramento a ser instalada na bacia, enquanto, a estação S2, localizada no centro da cidade de Poços de Caldas onde o corpo d'água possui baixa vazão e muitos lançamentos pontuais e difusos, se mostrou a mais impactada dentre as estações estudas apresentando baixos níveis de oxigênio dissolvido e altos valores de condutividade.

\section{AGRADECIMETOS}

Ao CNPq pelo auxilio concedido através de bolsa de iniciação científica (PIBIC/CNPq/UNIFAL-MG).

\section{REFERÊNCIAS}

ALVES, E. C.; SILVA, C. F.; COSSICH, E.S., TAVAVES, C.R.G.; SOUZA FILHO, E.E.; CARNIEL, A. Avaliação da qualidade da água da bacia do rio Pirapó - Maringá, Estado do Paraná, por meio de parâmetros físicos, químicos e microbiológicos. Acta Scientiarum Technology, Maringá (PR), v. 30, n. 1, p. 39-48, 2008.

AMERICAN PUBLIC HEALTH ASSOCIATION. Standard methods for the examination of water and wastewater. Byrd Prepress Springfield, Washington, DC. 21th ed, 2005. 1200 p.

BRIGANTE, J.; ESPÍNDOLA, E.L.G.; PORINELLI, J.; ELER, M. N. ; DORNFELD, C. B.; SILVA, M. R. C. Avaliação Ambiental do Rio Mogi-Guaçu: resultado de uma pesquisa com abordagem ecossistêmica. São Paulo: Rima, 2003. 57 p.

COSTA, P.C.G. ; DELGADO, S.L.; CARMOS, J.C.C. Projeto Hidrogeoambiental das Estâncias Hidrominerais da Companhia Mineradora de Minas Gerais - COMIG - Estância Hidromineral de Poços de Caldas. Relatório Técnico COMIG/Fundação Gorceix. 2001. 69 p.

ESPÍNDOLA, E.L.G. A bacia hidrográfica do rio Monjolinho: uma abordagem ecossistêmica e a visão interdisciplinar. RiMA, São Carlos, 2000. 188 p.

EYRE, B.D.; PEPPERELL, P. A spatialyy intensive approach to water quality monitoring in the Rous River catchment, NSW, Australia. Journal Environmental Management, v.56, p. 97-118, 1999.

FERNANDES, M.R. Poços de Caldas, Caracterização de Ecossistemas. Empresa de Assistência Técnica e Extensão Rural do Estado de Minas Gerais - EMATER-MG - Relatório Técnico. 2003. $56 \mathrm{p}$.

FREITAS, E.P; PECHE FILHO, A.; LEITE, J.F.M. Influencia da urbanização na bacia do rio Jundiaí-Mirim nas áreas de deságue e no risco de deslizamentos. In: XIX SIMPÓSIO 
BRASILEIRO DE RECURSOS HÍDRICOS, 2011, Maceió (AL), p. 202-202, 2011.

GOLTERMAN, H.L; CLYMO, R.S.; OHMSTAD, M.A.M. Methods for physical and chemical analysis of freshwaters. Oxford, Blackwell, 1978, 214p.

HUFSCHMIDT, M.M.; MCCAULEY, D.S. Water resources management planning and implementation. In: HASHIMOTO, M.; BARRET, B.F.D. (eds). Guidelines on Lake Management. Vol. 2. ILEC Foudation, Otsu, Japão.1991. p 53-81.

IBGE - INSTITUTO BRASILEIRO DE GEOGRAFIA E ESTATÍSTICA. Contagem da população. Disponível em: < http://www.ibge.gov.br/cidadesat> Acesso em: 11/10/2014.

MAASDAM, R.; SMITH, D.G. New Zealand's national river water quality network 2.

Relationships between physic-chemical data and environmental factors. New Zealand Journal of Marine and Freshwater Research, Wellington, v. 28, n. 1 p. 37-54, 1994.

MARGALEF, R. Limnologia. Barcelona, Ediciones Omega, 1983.1010 p.

MEYBECK, M.; CHAPMAN, D.V.; HELMER, P. Global freshwater quality. A first assessment. Oxford. World Health Organization. Blackwell, 1989. 356 p.

MORAESA, D.S.L.; JORDÃO, B.Q. Degradação de recursos hídricos e seus efeitos sobre a saúde humana. Revista Saúde Pública. Corumbá- Ms, v.3, n.363: p.370-374, mar. 2002.

MOTA, S. Introdução a Engenharia Ambiental. ABES - Associação Brasileira de Engenharia Sanitária e Ambiental, Rio de Janeiro, 1997. 419 p.

POÇOS DE CALDAS -. Mapa das bacias hidrográficas do município de Poços de Caldas, escala 1:10.000. Secretaria de Planejamento. Poços de Caldas, 2003.

ROCHA, O; PIRES, J.S.R.; SANTOS, J.E., A bacia hidrográfica como unidade de estudo e planejamento. In: Espíndola, E.L.G. (org.). A bacia hidrográfica do rio Monjolinho: uma abordagem ecossistêmica e a visão interdisciplinar. RiMA, São Carlos. 2000, pp.1-16

TAVARES, A.R. Monitoramento da qualidade das águas do rio Paraíba do Sul e diagnóstico de conservação. 2005. 176f. (Dissertação). Mestrado do Instituto Tecnológico de Aeronáutica ITA, São José dos Campos, São Paulo. 2005.

TUNDISI, J.G. Água no Século XXI: Enfrentando a Escassez. 2 ed. RiMA, São Carlos, 2005. $256 \mathrm{p}$.

TUCCI, C.E.M.; CLARKE, R.T. Impacto das mudanças da cobertura vegetal no escoamento. Revista Brasileira de Recursos Hídricos. Porto Alegre (RS), v.2, n.1: p.135-152, 1997.

TUCCI, C.E.M. Inundações urbanas na América do sul: águas urbanas. Porto Alegre: Associação Brasileira de Recursos Hídricos, 2003. 150 p. 
VEGA, M., PARDO, R., ENRIQUE, B.; DEBÁN, L. Assessment of seasonal and polluting effects on the quality of river water by exploratory data analysis. Water Research, Oxford, v. 32, n.12, p. 3581- 3592, 1998.

WORLD HEALTH ORGANIZATION. Guidelines for safe recreational water environments coastal and fresh waters. v.1. Geneva, Switzerland, 2003. 253p. 